\title{
Cytokines and dietary energy restriction in stable chronic obstructive pulmonary disease patients
}

\author{
I. Godoy*, Á.O. Campana*, R.R.C. Geraldo*, C.R. Padovani*, S.A.R Paiva*
}

Cytokines and dietary energy restriction in stable chronic obstructive pulmonary disease patients. I. Godoy, A.O. Campana, R.R.C. Geraldo, C.R. Padovani, S.A.R Paiva. (C) ERS Journals Ltd 2003.

ABSTRACT: Tumour necrosis factor (TNF)- $\alpha$ has been found to be increased in malnourished chronic obstructive pulmonary disease (COPD) patients; however, the main cause of this phenomenon remains undetermined. In normal subjects, TNF-a production may be induced by dietary energy deprivation. The aim of this study was to investigate if stable COPD patients present alterations of inflammatory mediators after $48 \mathrm{~h}$ of dietary energy restriction.

Fourteen COPD patients were admitted to the hospital while receiving an experimental diet with an energy content of approximately one-third of their energy needs. Clinical evaluation, nutritional assessment and serum levels of interleukin (IL)-6, TNF- $\alpha$ and C-reactive protein, and secretion of TNF- $\alpha$ by peripheral blood monocytes were assessed on admission and after the experimental diet. For reference values of the laboratory parameters, blood was collected from 10 healthy, elderly subjects.

COPD patients showed significantly higher serum concentrations of IL-6 than control subjects, however, the experimental diet was not associated with statistically significant changes in the inflammatory mediators.

The findings of this study, although preliminary because of the limited degree and duration of the energy restriction, suggest that the elevated levels of tumour necrosis factor- $\alpha$, previously described in undernourished or weight-losing chronic obstructive pulmonary disease patients, may not be linked to a decrease of dietary energy intake. Eur Respir J 2003; 22: 920-925.
*Faculdade de Medicina de Botucatu and \#Instituto de Biociências, Universidade Estadual Paulista (UNESP), São Paulo, Brazil.

Correspondence: I. Godoy, Faculdade de Medicina de Botucatu, Universidade Estadual Paulista (UNESP), Botucatu, São Paulo, Brazil 18618-000

Fax: 551438822238

E-mail: irma@fmb.unesp.br

Keywords: Chronic obstructive pulmonary disease

cytokines

energy restriction

interleukin-6

malnutrition

tumour necrosis factor- $\alpha$

Received: March 62003

Accepted after revision: August 132003

Supported by a grant from "Fundação de Amparo à Pesquisa do Estado de São Paulo" (FAPESP 95/0605-0) - FAPESP, São Paulo, Brazil.
Malnutrition is a common problem in many patients with chronic obstructive pulmonary disease (COPD), negatively affecting their exercise performance, health-related quality of life and survival rates [1]. Several studies confirm the presence of inflammatory mediators represented by elevated concentrations of acute-phase proteins, tumour necrosis factor (TNF)- $\alpha$ and its soluble receptors, in the peripheral blood of malnourished COPD patients [2-4]. However, the cause of systemic inflammation in malnourished or in weight-losing COPD patients remains unknown.

Several factors that can affect airway inflammation in COPD patients have been described [5]. Chronic bacterial colonisation can contribute significantly to higher levels of inflammatory mediators, even in clinically stable COPD patients [5]. Another explanation for the elevated serum concentration of inflammatory mediators may be chronic or even intermittent hypoxaemia. TAKABATAKE et al. [6] found an inverse correlation between arterial oxygen tension $\left(\mathrm{Pa}, \mathrm{O}_{2}\right)$, circulating TNF- $\alpha$ and soluble TNF- $\alpha$ receptor (sTNFR) 75 and suggested that systemic hypoxaemia in patients with COPD is associated with activation of the TNF- $\alpha$ system in vivo.

Increased TNF- $\alpha$ production by peripheral blood monocytes (PBMC) has also been reported in healthy subjects during severe calorific deprivation [7], and suppression of the elevated production has been observed during the refeeding process [8]. In a previous study, higher TNF- $\alpha$ production by PBMC of COPD patients with recent weight loss was shown, in contrast with monocytes of weight-stable COPD and control subjects [9]. The findings suggested a possible role for TNF- $\alpha$ production as a marker of a weight-losing process in these patients. However, the change of TNF- $\alpha$ production could also serve to identify a second pathophysiological process, such as altered dietary intake, which may be found in malnourished patients. Therefore, it was hypothesised that dietary energy restriction induces modifications of inflammatory markers in COPD patients. Here, a study evaluating the effect of a 48-h period of dietary energy restriction on the serum levels of TNF- $\alpha$ and interleukin (IL)- 6 , and on TNF- $\alpha$ secretion by PBMC, is reported in clinically and weight-stable nonhypoxaemic COPD patients.

\section{Subjects and methods}

\section{Subjects}

This study was approved by the Medical Ethics Committee of the Botucatu Medical School and informed consent was obtained from all the subjects who participated in the investigation. The study group consisted of 14 COPD patients seen consecutively at the Pulmonary Outpatient Clinic of the authors' hospital. Patients were included if they fulfilled the criteria for COPD according to the American Thoracic Society (ATS) guidelines [10]. Furthermore, the forced expiratory volume in one second (FEV1) had to be $<70 \%$ of the reference value and the increase in FEV1, after inhalation of a 
$\beta_{2}$-agonist, $<10 \%$ of the reference value. Only patients in a clinically stable condition without hypoxaemia and evidence of weight loss during the last year, with no recorded infections, exacerbation of respiratory symptoms, changes in medications 2 months before the study and without clinical signs of oedema were included in the study. Patients with concomitant confounding diseases such as malignant disorders, gastrointestinal abnormalities, recent surgery or severe endocrine disorders were excluded.

Ten healthy, elderly subjects, aged 48-68 yrs, paired for smoking habits with COPD patients at the time of study and free of lung disease, acted as a control group for inflammatory mediator determinations. Baseline characteristics of COPD patients and control subjects are presented in table 1 .

\section{Study design}

All COPD patients who agreed to participate in the study were admitted to the hospital. Their usual dietary intake was assessed and energy needs were calculated on admission. Both before and after the experimental diet, all subjects were measured anthropometrically and tested for body composition with bioelectric impedance analysis. Venous blood $(45 \mathrm{~mL})$ was obtained by venipuncture in vacuum tubes (Vacuteiner; Becton Dickinson, Franklin Lakes, NJ, USA) containing heparin for monocyte isolation. For serum measurements, blood was collected in vacuum tubes containing serum separator gel (Vacuteiner; Becton Dickinson). Patients received an experimental diet with an energy content of one-third of their energy needs during $48 \mathrm{~h}$. The measurements were performed early in the morning $(07: 00 \mathrm{~h})$, when patients were in the fasting state for $\geqslant 10 \mathrm{~h}$.

\section{Experimental diet}

Individual energy need was calculated according to the Harris-Benedict equation [11]. The obtained value was multiplied by the activity factor (1.2), and then by the injury factor (1.3). Each patient was fed with a personally designed diet containing one-third of their calculated energy need during 2 days. The energy content of the diet was $\left(\right.$ mean \pm SD) $685 \pm 60 \mathrm{kcal} \cdot \mathrm{day}^{-1}$. This diet had the following macronutrient composition: protein $38 \pm 4 \%$, fat $21 \pm 6 \%$ and carbohydrate $42 \pm 5 \%$.

\section{Lung function}

FEV1 and forced vital capacity were calculated from the flow/volume curve using a spirometer (Med-Graph 1070; Medical Graphics Corporation, St Paul, MN, USA),

Table 1.-Characteristics of the study subjects

\begin{tabular}{lcc}
\hline & Control subjects & COPD \\
\hline Subjects n & 10 & 14 \\
Age yrs & $54(52-58)$ & $62(59-68)$ \\
M/F & $5 / 5$ & $14 / 0$ \\
Current smokers yes/no & $4 / 6$ & $5 / 9$ \\
FEV $1 \%$ & $108.6 \pm 8.7$ & $43.9 \pm 16.9$ \\
$P a, \mathrm{O}_{2} \mathrm{kPa}$ & $10.7 \pm 1.3$ & $9.8 \pm 0.7$ \\
\hline
\end{tabular}

Data represent mean \pm SD or median (quartiles 1-3) unless otherwise stated. COPD: chronic obstructive pulmonary disease; M: male; F: female; FEV1: forced expiratory volume in one second; $\mathrm{Pa}, \mathrm{O}_{2}$ : arterial oxygen tension. according to the criteria set by ATS [12]. Lung function values were expressed as per cent of predicted [13]. Blood was drawn from the brachial artery at rest while breathing room air. $\mathrm{Pa}, \mathrm{O}_{2}$ and arterial carbon dioxide tension were analysed on a blood analyser (Stat Profile 5 Plus; Nova Biomedical, Whaltham, MA, USA).

\section{Dietary intake}

An estimate of the usual daily nutrient intake during the past 6 months was obtained from a food-frequency questionnaire [14]. The instrument listed 120 foods and beverage items. The information was coded for computer nutrient analysis by the same trained dietician. Dietary information was converted to energy using the computer software "Programa de Apoio à Nutrição" (Centro de Informática em Saúde da Escola de Medicina, São Paulo, Brazil).

\section{Body composition}

The anthropometric nutritional assessment consisted of height and body weight with estimation of the percentage of ideal body weight (IBW) according to the Metropolitan Life Insurance Tables [15]. Body composition was estimated using single-frequency $(50 \mathrm{kHz})$ bioelectrical impedance analysis (BIA 101; RJL Systems, Detroit, MI, USA) while subjects were in a supine position. Fat-free mass (FFM) was calculated using the disease-specific equation of SCHOLS et al. [16]. Fat mass (kg) was calculated by subtracting FFM from body weight.

\section{Monocyte isolation and culture}

PBMC were isolated from heparinised blood drawn between 07:00-08:00 h using a density gradient, followed by plastic adherence as described elsewhere [9]. In short, PBMC were isolated from heparinised blood using Histopaque 1077 (Sigma Chemical Co., St Louis, MO, USA), washed three times with Hanks' solution (Sigma Chemical Co.). Cell suspensions were prepared with PBMC at $2 \times 10^{6}$ cells $\cdot \mathrm{mL}^{-1}$ in Roswell Park Memorial Institute (RPMI)-1640 medium (Sigma Chemical Co.), supplemented with $5 \%$ heat-inactivated autologus serum, $2 \mathrm{mM}$ glutamine, $100 \mathrm{U} \cdot \mathrm{mL}^{-1}$ penicillin and $10 \mathrm{mM}$ HEPES $(N$-2-hydroxyethylpiperazine- $N$-2-ethanesulphonic acid) buffer (that hereafter will be referred as $\mathrm{mRPMI}$ ), and incubated for $2 \mathrm{~h}$ in a humidified atmosphere of $5 \% \mathrm{CO}_{2}$ at $37^{\circ} \mathrm{C}$. Nonadherent cells were removed by three washes with plain RPMI and counted. The adherents cells were refed with mRPMI at a volume of $1 \mathrm{~mL} \cdot 1 \times 10^{6}$ cells $^{-1}$ with and without lipopolysaccharide (LPS; Escherichia coli serotype O55:B5; Sigma Chemical Co.) at a final concentration of $100 \mathrm{ng} \cdot \mathrm{mL}^{-1}$ and incubated for $24 \mathrm{~h}$. Supernatants were collected, centrifuged, and stored at $-80^{\circ} \mathrm{C}$ until cytokine analysis. After harvesting the supernatants, adherents cells were stained with nonspecific esterase staining (Sigma Chemical Co.), and cell viability was determined using a trypan blue exclusion test. Adherent cells contained $>95 \%$ monocytes; $>98 \%$ were viable.

\section{Interleukin-6 and tumour necrosis factor- $\alpha$ measurements}

IL-6 and TNF- $\alpha$ in monocyte supernatant and serum were measured in duplicate using sandwich enzyme-linked immunosorbent assay (ELISA) kits (Biosource International, Camarillo, CA, USA). Briefly, standards and samples were 
added to individual wells in microtitre strips coated with antibody specific for the cytokine being measured. After several washes to remove unbound proteins, an enzymelinked biotin conjugate was added to the wells. Following several additional washes to remove unbound antibodyenzyme reagent, an enzyme solution (streptavidin-peroxidase) was added. After a period of incubation and additional washes to remove all the unbound enzyme, a substrate solution (tetramethylbenzidine) was added. The reaction was stopped by adding an acidic solution. Absorbance was measured spectrophotometrically at $450 \mathrm{~nm}$ using a micro-ELISA autoreader. The lower and upper limits of detection for TNF$\alpha$ were 0.5 and $32 \mathrm{pg} \cdot \mathrm{mL}^{-1}$ (human TNF- $\alpha$ US-cytoscreen) or 1 and $1,000 \mathrm{pg} \cdot \mathrm{mL}^{-1}$ (human TNF- $\alpha$ cytoscreen); for IL-6 they were 0.16 and $10 \mathrm{pg} \cdot \mathrm{mL}^{-1}$ (human IL-6 US-cytoscreen) or 2 and $500 \mathrm{pg} \cdot \mathrm{mL}^{-1}$ (human IL-6 cytoscreen).

\section{C-reactive protein}

C-reactive protein (CRP) was measured by immunonephelemetry (Behring Diagnostics Inc., Westowood, MA, USA).

\section{Statistical analyses}

When there was a normal distribution, comparisons between COPD patients and control subjects were made by an unpaired t-test. Difference within the group before and after experimental diet was analysed using a paired t-test. When variables were not normally distributed, comparisons between two groups were made with a Mann-Whitney U-test. The Wilcoxon test was used to analyse differences within the group before and after experimental diet. Differences in dichotomous variables were statistically compared using the Chi-squared or Fisher test. Repeated-measure analysis of variance, followed by the Tukey test, was used to study differences among usual dietary intake, energy needs and dietary intake during the experimental diet. To analyse interrelations between variables, Pearson's or Spearman's correlation coefficients were calculated.

\section{Results}

\section{Dietary intake and body composition}

Values for IBW were significantly higher in control subjects (mean \pm SD $118 \pm 18 \%$ ) when compared with COPD patients $(98 \pm 13 \%, \mathrm{p}=0.005) ; 28.5 \%$ of COPD patients and $10 \%$ of control subjects presented with an IBW of $<90 \%(\mathrm{p}>0.05)$.

Usual daily energy intake $\left(47.5 \pm 15.0 \mathrm{kcal} \cdot \mathrm{kg}^{-1} \cdot \mathrm{day}^{-1}\right)$ in COPD patients was significantly higher than the calculated energy needs $\left(33.1 \pm 3.8 \mathrm{kcal} \cdot \mathrm{kg}^{-1} \cdot \mathrm{day}^{-1}\right)$ and both values were significantly higher than the dietary intake during experimental diet $\left(11.0 \pm 1.4 \mathrm{kcal} \cdot \mathrm{kg}^{-1} \cdot \mathrm{day}^{-1}\right)$. Values for body composition variables before and after the experimental diet are shown in table 2. After experimental diet, patients lost $1.36 \pm 0.58 \mathrm{~kg}$, reflected mainly by a decrease in FFM.

\section{Inflammatory markers in patients with chronic obstructive pulmonary disease and healthy subjects}

Inflammatory markers were compared between patients and control subjects as shown in table 3. IL-6 serum concentrations were significantly increased in COPD patients $(p<0.05)$. No significant differences were seen in serum and
Table 2.-Body composition before and after experimental diet

\begin{tabular}{lccc}
\hline & Before & After & p-value \\
\hline Subjects n & 14 & 14 & \\
Weight kg & $62.8 \pm 6.9$ & $61.4 \pm 6.9$ & $<0.001$ \\
Fat mass kg & $18.3 \pm 5.6$ & $18.7 \pm 5.1$ & 0.178 \\
Fat-free mass kg & $44.5 \pm 3.6$ & $42.7 \pm 3.4$ & 0.001 \\
\hline
\end{tabular}

Data represent mean \pm SD unless otherwise stated.

LPS-stimulated cell culture supernatant TNF- $\alpha$ between patients and healthy controls. TNF- $\alpha$ in monocyte supernatant of spontaneous cell culture was not detectable with this assay. Serum CRP levels tended to be higher in COPD and was positively related to IL-6 $(\mathrm{r}=0.73, \mathrm{p}=0.00076)$; seven patients presented values $>5 \mathrm{mg} \cdot \mathrm{L}^{-1}$. None of the values for the inflammatory mediators correlated with values of body composition, dietary intake, lung function and arterial blood gases.

\section{Inflammatory markers in chronic obstructive pulmonary disease patients before and after the experimental diet}

Comparison of inflammatory markers before and after $48 \mathrm{~h}$ of experimental diet are shown in table 4 and figure 1 . The experimental diet did not induce statistically significant changes in serum levels of CRP, IL-6, TNF- $\alpha$ and in LPSstimulated TNF- $\alpha$ secretion by monocytes.

Table 3.-Inflammatory markers in patients with chronic obstructive pulmonary disease (COPD) compared with control subjects

\begin{tabular}{lccc}
\hline & $\begin{array}{c}\text { Control } \\
\text { subjects }\end{array}$ & $\begin{array}{c}\text { COPD } \\
\text { subjects }\end{array}$ & p-value \\
\hline Subjects n & 10 & 14 & \\
CRP mg $\cdot \mathrm{dL}^{-1}$ & $1.7(0.9-2.1)$ & $5.6(1.0-20.2)$ & 0.095 \\
Serum TNF- $\alpha \mathrm{pg} \cdot \mathrm{mL}^{-1}$ & $2.0(1.6-3.2)$ & $2.0(1.6-2.8)$ & 0.953 \\
Serum IL-6 pg $\cdot \mathrm{mL}^{-1}$ & $1.6(0.4-14)$ & $3.7(2.6-4.8)$ & 0.038 \\
LPS-TNF- $\alpha \mathrm{pg} \cdot 10^{6} \mathrm{cell}^{-1}$ & $2957 \pm 1512$ & $2227 \pm 1540$ & 0.55 \\
\hline
\end{tabular}

Data represent mean \pm SD or median (quartiles 1-3) unless otherwise stated. CRP: C-reactive protein; TNF: tumour necrosis factor; IL: interleukin; LPS-TNF- $\alpha$ : lipopolysaccharide-stimulated cell culture supernatant TNF- $\alpha$.

Table 4.-Markers of inflammation before and after experimental diet

\begin{tabular}{lccc}
\hline & \multicolumn{2}{c}{ COPD subjects } & \multirow{2}{*}{ p-value } \\
\cline { 2 - 3 } & Before & After & \\
\hline Subjects n & 14 & 14 & \\
CRP mg $\cdot \mathrm{L}^{-1}$ & $5.6(1.0-20.2)$ & $6.8(3.7-12.8)$ & 0.32 \\
SerumTNF- $\alpha \mathrm{pg} \cdot \mathrm{mL}^{-1}$ & $2.0(1.6-2.8)$ & $2,1(1.3-3.9)$ & 0.301 \\
Serum IL-6 pg $\cdot \mathrm{mL}^{-1}$ & $3.7(2.6-4.8)$ & $3.2(1.8-4.4)$ & 0.241 \\
LPS-TNF- $\alpha \mathrm{pg} \cdot 10^{6}$ cell $^{-1}$ & $2227 \pm 1540$ & $2333 \pm 1075$ & 0.791
\end{tabular}

Data represent mean \pm SD or median (quartiles 1-3) unless otherwise stated. COPD: chronic obstructive pulmonary disease; CRP: C-reactive protein; TNF: tumour necrosis factor; IL: interleukin; LPS-TNF- $\alpha$ : lipopolysaccharide-stimulated cell culture supernatant TNF- $\alpha$. 

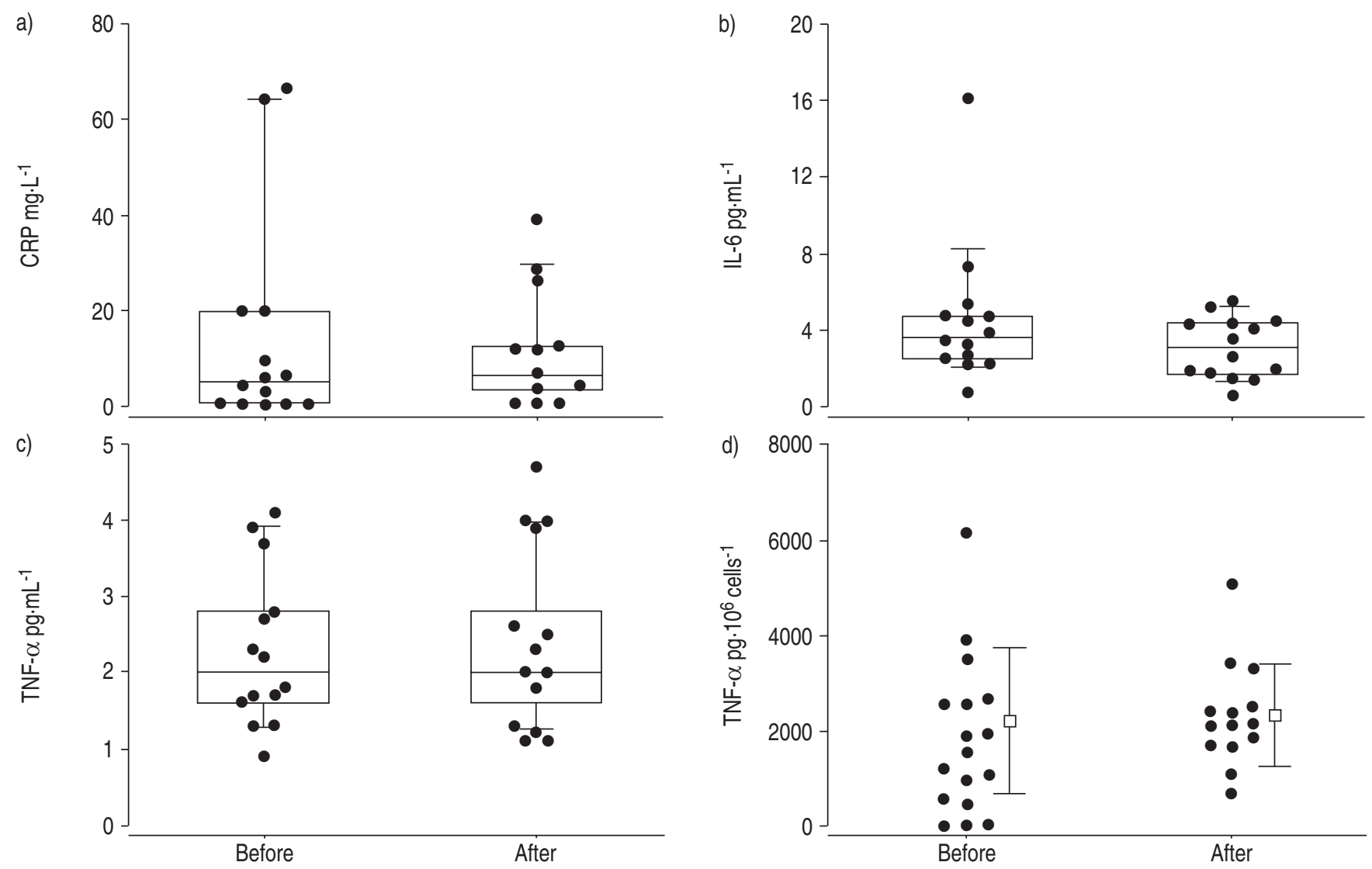

Fig. 1. - Individual plots $(\bullet)$ before and after the experimental diet for a) C-reactive protein (CRP), b) serum interleukin (IL)-6, and c) serum tumour necrosis factor (TNF)- $\alpha$. The box indicates the 25th to 75th percentiles and the horizontal line indicates the median value. Whiskers below and above the box indicate the 10th and 90th percentiles, respectively. d) Lipopolysaccharide-stimulated cell culture supernatant TNF- $\alpha$ before and after the experimental diet. The mean $(\square) \pm$ SD values are also shown.

\section{Discussion}

The purpose of this study was to investigate the effect of energy restriction on the activation of inflammatory systems in patients with COPD. Clinically stable COPD patients with a moderate/severe degree of airway obstruction without evidence of hypoxaemia and/or modifications of body weight during the last year were submitted to energy dietary restriction over $48 \mathrm{~h}$. During the period of experimental diet, they ingested an energy content of $\sim 33 \%$ of their estimated energy needs or $27 \%$ of their usual energy ingestion, resulting in a significant weight loss without modification of inflammatory markers.

In these patients, the prevalence of nutritional depletion, a dietary intake significantly higher than estimated energy needs and evidence suggesting ongoing inflammatory reaction, as shown by the finding of higher serum IL-6 concentration, are in agreement with previously available data obtained in clinically stable COPD patients [1, 17-20]. Although no significant relationship could be established, these findings may indicate that the higher levels of IL- 6 are not related to a deficit of energy intake and that other factors may contribute to the elevated levels of this cytokine in COPD patients.

COPD patients studied in the present investigation were weight stable during the last year and presented serum concentration and secretion of TNF- $\alpha$ by PBMC that was not significantly different when compared with values obtained in control healthy subjects. This is in agreement with previous results obtained by DI FRANCIA et al. [2] in COPD patients weighing more than the lower limit of normal according to the Metropolitan Life Insurance Company tables [15], and by the present authors in patients with stable weight [9]. However, higher serum concentrations of TNF- $\alpha$, of sTNFRs (TNFR75 and TNFR55) and secretion of TNF- $\alpha$ by PBMC have been described in COPD patients showing an unintentional weight loss, a body weight less than the lower limit of normal [2], a body weight fluctuation of $>5 \%$ during the last year [9], and also in patients with increased resting energy expenditure, decreased FFM, body mass index and creatinineheight index [3, 21]. These studies support a hypothesis linking inflammatory and catabolic states in COPD patients.

The cause of the systemic inflammation in malnourished COPD patients is unknown; various factors that may interfere with cachexia and inflammatory markers have been considered [5, 6, 22]. TAKABATAKE et al. [6] found an inverse correlation between $\mathrm{Pa}, \mathrm{O}_{2}$ and circulating TNF- $\alpha$ and sTNFR levels in patients with COPD, suggesting that systemic hypoxaemia may be associated with activation of the TNF$\alpha$ system in vivo [6]. Another explanation for elevated serum concentration of inflammatory mediators may be some factor related to an altered dietary intake [8].

The effects of dietary intake fluctuation on inflammatory mediators have been examined in clinical entities of different aetiologies, pathophysiological mechanisms of malnutrition, type and duration of dietary intake fluctuation, baseline nutritional state and also whether the tests were made during dynamic weight loss or after weight stabilisation [7, 8, 23]. Data obtained in healthy subjects, who underwent severe calorific deprivation, and in weight-losing and weight-stable patients with anorexia nervosa, suggested that increased levels of TNF- $\alpha$ are related to decreased energy ingestion and that active weight loss should be present to stimulate secretion of 
cytokines [7, 8]. This is in agreement with findings of a previous study, where it was found that COPD patients with active weight loss presented with a significantly higher TNF- $\alpha$ secretion by PBMC when compared with weight-stable patients and control subjects. In the present study, it was also shown that at 6 months follow-up, in the absence of ongoing weight loss, the difference in TNF- $\alpha$ secretion between groups was not maintained [9].

In contrast with the suggestions of the previous abovementioned studies [7-9], the energy dietary restriction, over $48 \mathrm{~h}$, was not associated with significant changes in serum concentrations of IL-6, CRP and TNF- $\alpha$ and in the secretion of TNF- $\alpha$ by PBMC. However, the degree and duration of energy restriction may be responsible by the absence of the increased inflammatory response during the experiment. Although the energy intake of the patients during the experimental period $\left(685 \pm 60 \mathrm{kcal} \cdot \mathrm{day}^{-1}\right)$ was not as low as that provided by VAISMAN et al. [7] to healthy subjects $\left(100 \mathrm{kcal} \cdot \mathrm{day}^{-1}\right)$, it was lower than the energy intake reported in anorexia nervosa patients $\left(800-1,300 \mathrm{kcal}^{\left.-\mathrm{day}^{-1}\right)}\right.$ [8] and also during periods of disease exacerbation in COPD patients $\left(1,350 \mathrm{kcal} \cdot\right.$ day $\left.^{-1}\right)[24,25]$. In this study, the experimental diet led to alterations of body weight $(1.36 \pm 0.58 \mathrm{~kg})$ and body composition, reflected mainly by decreased FFM that can be accounted for, at least in part, by body hypohydration and calorific deficit. These findings are similar to those observed during the acute phase of starvation experiments when the cumulative weight loss varied 1.56-2.09 kg, after $48 \mathrm{~h}$ of $500 \mathrm{kcal} \cdot \mathrm{day}^{-1}$ intake attributed mainly to water loss during early starvation [26, 27]. Therefore, the restriction of energy intake, to which the COPD patients were subjected, appears significant.

To date, there are no available data concerning the period of time needed to stimulate an inflammatory response in COPD patients. It could be hypothesised that a longer period of energy restriction may have elicited an inflammatory response, but, the patients could not be subjected to any additional nutritional risk. Exacerbation of COPD has been associated with increased airway and systemic inflammation $[28,29]$. The underlying mechanisms inducing and controlling the inflammatory process during periods of disease exacerbation are far more complex than the energy restriction induced in this study $[5,28,29]$. However, as decreased dietary intake has been described during these periods $[24,25]$ it cannot be completely excluded that, at least in part, low energy intake may contribute to the increased inflammatory status. Therefore, in the present work, the design involved the use of a period of low energy intake similar to that observed in disease exacerbation periods [24, 30]. During these periods, the dietary intake is significantly lower (mean 43\%) than the habitual dietary intake of the period prior to admission to the hospital, followed by significant increase of intake on the second day after admission. Thereafter, the dietary intake improved quickly for the remaining hospitalisation period $[24,30]$.

In summary, this study aimed to examine the effect of energy restriction and its immediate consequences in the inflammatory status in chronic obstructive pulmonary disease patients. It was shown that a decrease of energy intake for short periods of time did not activate the tumour necrosis factor- $\alpha$ and interleukin- 6 systems in clinically and weightstable chronic obstructive pulmonary disease patients. The findings of the study, although preliminary because of the limited degree and duration of the energy restriction, suggest that the elevated serum levels or secretion of tumour necrosis factor- $\alpha$ previously described in undernourished or weight-losing chronic obstructive pulmonary disease patients may not be linked to a decrease of dietary energy intake in these patients.

\section{References}

1. Wouters EFM, Creutzberg EC, Schols AMWJ. Systemic effects in COPD. Chest 2002; 121: 127S-130S.

2. Di Francia M, Barbier D, Mege JL, Orehek J. Tumor necrosis factor-alpha levels and weight loss in chronic obstructive pulmonary disease. Am J Respir Crit Care Med 1994; 150: 1453-1455.

3. Schols AMWJ, Buurman WA, Brekel AJS, Dentener MA, Wouters EFM. Evidence for a relation between metabolic derangements and increased levels of inflammatory mediators in a subgroup of patients with chronic obstructive pulmonary disease. Thorax 1996; 51: 819-824.

4. Creutzberg EC, Schols AMWJ, Welling-Scheepers CAPM, Buurman WA, Wouters EFM. Characterization of nonresponse to high caloric oral nutritional therapy in depleted patients with chronic obstructive pulmonary disease. Am J Respir Crit Care Med 2000; 161: 745-752.

5. Hill A, Gompertz S, Stockley R. Factors influencing airway inflammation in chronic obstructive pulmonary disease. Thorax 2000; 55: 970-977.

6. Takabatake N, Nakamura $\mathrm{H}$, Abe $\mathrm{S}$, et al. The relationship between chronic hypoxemia and activation of the tumor necrosis factor- $\alpha$ system in patients with chronic obstructive pulmonary disease. Am J Respir Crit Care Med 2000; 161: $1179-1184$

7. Vaisman N, Schattner A, Hahn T. Tumor necrosis factor production during starvation. Am J Med 1989; 87: 115.

8. Vaisman N, Hahn T. Tumor necrosis factor- $\alpha$ and anorexia cause or effect? Metabolism 1991; 40: 720-723.

9. de Godoy I, Donahoe M, Calhoun WJ, Mancino J, Rogers RM. Elevated TNF-alpha production by peripheral blood monocytes of weight-losing COPD patients. Am J Respir Crit Care Med 1996; 153: 633-637.

10. American Thoracic Society. Standards for the diagnosis and care of patients with chronic obstructive pulmonary disease. Am J Respir Crit Care Med 1995; 152: S77-S120.

11. Bursztein S, Elwyn DH, Askanazi J, Kinney JM, eds. Energy Metabolism, Indirect Calorimetry, and Nutrition. 1st Edn. Baltimore, Williams \& Wilkins, 1989.

12. American Thoracic Society. Standardization of spirometry 1987 update. Am Rev Respir Dis 1987; 136: 1285-1298.

13. Knudson RJ, Lebowitz MD, Holberg CJ, Burrows B. Changes in the normal maximal expiratory flow-volume curve with growth and aging. Am Rev Respir Dis 1983; 127: 725-734.

14. Willet W, ed. Nutritional Epidemiology. 2nd Edn. New York, Oxford University Press, 1998.

15. Metropolitan Life Insurance Company. New weight standards for men and women. Stat Bull 1983; 64: 1-9.

16. Schols AMWJ, Wouters EFM, Soeters PB, Westerterp KR Body composition by bioelectrical-impedance compared with deuterium dilution and skinfold anthropometry in patients with chronic obstructive pulmonary disease. Am J Clin Nutr 1991; 53: 421-424.

17. Paiva SA, Godoy I, Vannucchi H, Favaro RM, Geraldo RR, Campana AO. Assessment of vitamin A status in chronic obstructive pulmonary disease patients and healthy smokers. Am J Clin Nutr 1996; 64: 928-934.

18. Hunter AM, Carey MA, Larsh HW. The nutritional status of patients with chronic obstructive pulmonary disease. Am Rev Respir Dis 1981; 124: 376-381.

19. Ferroni P, Basili S, Alessandri C, et al. Proinflammatory cytokines and hemostatic system in patients with chronic obstructive pulmonary disease. Platelets 1997; 8: 255-259.

20. Yasuda N, Gotoh K, Minatoguchi S, et al. An increase of soluble Fas, an inhibitor of apoptosis, associated with progression of COPD. Respir Med 1998; 92: 993-999.

21. Eid AA, Ionescu AA, Nixon LS, et al. Inflammatory response and body composition in chronic obstructive pulmonary disease. Am J Respir Crit Care Med 2001; 164: 1414-1418.

22. Schols AMWJ, Creutzberg EC, Buurman WA, Campfield LA, 
Saris WHM, Wouters EFM. Plasma leptin is related to proinflammatory status and dietary intake in patients with chronic obstructive pulmonary disease. Am J Respir Crit Care Med 1999; 160: 1220-1226.

23. Doherty JF, Golden MHN, Remick DG, Griffin GE. Production of interleukin- 6 and tumour necrosis factor- $\alpha$ in vitro is reduced in whole blood of severely malnourished children. Clin Sci 1994; 86: 347-351.

24. Vermeeren MAP, Schols AMWJ, Wouters EFM. Effects of an acute exacerbation on nutritional and metabolic profile of patients with COPD. Eur Respir J 1997; 10: 2264-2269.

25. Saudny-Unterberger H, Martin JG, Gray-Donald K. Impact of nutritional support on functional status during an acute exacerbation of chronic obstructive pulmonary disease. Am J Respir Crit Care Med 1997; 156: 794-799.

26. Hoffer JL. Metabolic consequences of starvation. In: Shils ME, Olson JA, Shike M, Ross CA, eds. Modern Nutrition in Health and Disease. 9th Edn. Baltimore, Williams \& Wilkins, 1998; pp. 645-665.

27. Johnson HL, Consolazio CF, Krzywicki HJ, Isaac GJ, Witt NF. Metabolic aspects of caloric restriction: nutrient balances with 500-kilocalorie intakes. Am J Clin Nutr 1971; 24: 913-923.

28. Bhowmik A, Seemungal TAR, Spasford RJ, Wedzicha JA. Relation of sputum inflammatory markers to symptoms and lung function changes in COPD exacerbations. Thorax 2000; 55: $114-120$

29. Wedzicha JA, Seemungal TAR, MacCallum PK, et al. Acute exacerbations of chronic obstructive pulmonary disease are accompanied by elevations of plasma fibrinogen and serum IL-6 levels. Thromb Haemost 2000; 84: 210-215.

30. Creutzberg AC, Wouters EFM, Vanderhoven-Augustin IML, Dentener MA, Schols AMW. Disturbances in leptin metabolism are related to energy imbalance during acute exacerbations of chronic obstructive pulmonary disease. Am J Respir Crit Care Med 2000; 162: 1239-1245. 